\title{
Processos de extração e usos industriais de óleos de andiroba e açaí: uma revisão
}

\author{
Extraction processes and industrial uses of andiroba and açaí oils: a review \\ Procesos de extracción y usos industriales de los aceites de andiroba y açaí: una revisión
}

Recebido: 03/09/2021 | Revisado: 13/09/2021 | Aceito: 17/09/2021 | Publicado: 18/09/2021

\author{
Gilso Blanco Lira \\ ORCID: https://orcid.org/0000-0003-4902-9413 \\ Universidade Federal do Pará, Brasil \\ E-mail: gilsoblira@gmail.com \\ Anna Sylmara da Costa Lopes \\ ORCID: https://orcid.org/0000-0002-5769-5582 \\ Universidade Federal do Pará, Brasil \\ E-mail: annalopes.ta.qi@hotmail.com \\ Fabiana Cristina de Araújo Nascimento \\ ORCID: https://orcid.org/0000-0002-7641-2752 \\ Universidade Federal do Pará, Brasil \\ E-mail: fabiananascimento987@gmail.com \\ Gyselle dos Santos Conceição \\ ORCID: https://orcid.org/0000-0003-4746-6521 \\ Universidade Federal do Pará, Brasil \\ E-mail: gysa.com.y@hotmail.com \\ Davi do Socorro Barros Brasil \\ ORCID: https://orcid.org/0000-0002-1461-7306 \\ Universidade Federal do Pará, Brasil \\ E-mail: davibb@ufpa.br
}

\begin{abstract}
Resumo
A região amazônica apresenta uma grande diversidade de plantas ricas em compostos bioativos, cuja exploração racional contribui para o desenvolvimento sustentável da região. Dentre estas destacam-se a andiroba (Carapa guianenses) e o açaí (Euterpe oleracea), a partir dos quais se extraem óleos que possuem grande potencial para serem utilizados nas indústrias de cosméticos, farmacêutica, alimentícia e até mesmo na produção de combustíveis renováveis. Dessa forma, objetivou-se realizar uma pesquisa bibliográfica acerca dos métodos de extração dos óleos de andiroba e açaí, bem como suas aplicações na indústria e as novas novidades tecnológicas. Tais óleos são muito utilizados na medicina popular em virtude de suas inúmeras propriedades medicinais. $\mathrm{O}$ óleo de andiroba possui ação antiinflamatória, antimicrobiana, inseticida, etc., que são atribuídas principalmente a seu conteúdo de limonóides. Já o óleo de açaí apresenta uma grande quantidade de compostos fenólicos, como antocianinas e perfil de ácidos graxos benéficos à saúde, sendo muito utilizado como agente antioxidante, anticarcinogênico, combatendo doenças cardiovasculares e neurodegenerativas. Há inúmeros métodos utilizados para a extração desses óleos, entre estes, o artesanal, prensagem a frio, por fluído supercrítico, por solventes orgânicos, enzimático, etc. Mesmo assim, o método de prensagem a frio ainda é o mais utilizado pelas indústrias, uma vez que se obtém produtos de boa qualidade e preserva os seus compostos bioativos, bem como o mercado de cosméticos é o que mais utiliza esses óleos.
\end{abstract}

Palavras-chave: Óleo; Andiroba; Açaí; Métodos de extração; Aplicações industriais.

\begin{abstract}
The Amazon region has a great diversity of plants rich in bioactive compounds, whose rational exploitation contributes to the sustainable development of the region. Among these stand out andiroba (Carapa guianeses) and açaí (Euterpe oleracea), from which oils are extracted that have great potential to be used in the cosmetic, pharmaceutical, food and even in the production of renewable fuel. Thus, the objective was to carry out bibliographic research on the methods of extraction of andiroba and açai oils, as well as their applications in the industry and new technological innovations. Such oils are widely used in popular medicine due to their numerous medicinal properties. Andiroba oil has anti-inflammatory, antimicrobial, insecticide, etc., which are mainly attributed to its limonoid content. Açaí oil, on the other hand, has a large amount of phenolic compounds, such as anthocyanins and a profile of fatty acids that are beneficial to health, being widely used as an antioxidant, anticarcinogenic agent, fighting cardiovascular and neurodegenerative diseases. There are numerous methods used to extract these oils, including artisanal, cold pressing, supercritical fluid, organic solvents, enzymatic, etc. Even so, the cold pressing method is still the most used by industries, since it obtains better quality products and preserves their bioactive compounds, and the cosmetics market is the one that most uses these oils.
\end{abstract}

Keywords: Oil; Andiroba; Açaí; Extraction methods; Industrial applications. 


\begin{abstract}
Resumen
La región amazónica tiene una gran diversidad de plantas ricas en compuestos bioactivos, cuya explotación racional contribuye al desarrollo sustentable de la región. Entre estos destacan la andiroba (Carapa guianense) y el açaí (Euterpe oleracea), de los cuales se extraen aceites que tienen un gran potencial para ser utilizados en la cosmética, farmacéutica, alimentaria e incluso en la producción de combustibles renovables. Así, el objetivo fue realizar una investigación bibliográfica sobre los métodos de extracción de los aceites de andiroba y açaí, así como sus aplicaciones en la industria y las nuevas innovaciones tecnológicas. Dichos aceites se utilizan ampliamente en la medicina popular debido a sus numerosas propiedades medicinales. El aceite de andiroba tiene propiedades antiinflamatorias, antimicrobianas, insecticidas, etc., que se atribuyen principalmente a su contenido de limonoides. El aceite de Açaí, por su parte, tiene una gran cantidad de compuestos fenólicos, como las antocianinas y un perfil de ácidos grasos beneficiosos para la salud, siendo ampliamente utilizado como antioxidante, agente anticancerígeno, combatiendo enfermedades cardiovasculares y neurodegenerativas. Existen numerosos métodos utilizados para extraer estos aceites, incluyendo artesanal, prensado en frío, fluido supercrítico, solventes orgánicos, enzimáticos, etc. Aun así, el método de prensado en frío sigue siendo el más utilizado por las industrias, ya que obtiene productos de mejor calidad y conserva sus compuestos bioactivos, siendo el mercado de la cosmética el que más utiliza estos aceites.
\end{abstract}

Palabras clave: Aceite; Andiroba; Açaí; Métodos de extracción; Aplicaciones industriales.

\title{
1. Introdução
}

A região amazônica é uma importante fonte de espécies vegetais produtoras de sementes oleaginosas, das quais se extraem óleos vegetais de composição química e propriedades físico-químicas variadas e que apresentam potencial econômico, tecnológico e nutricional, despertando o interesse do meio científico em diversificadas áreas, como na fabricação de produtos farmacêuticos, cosméticos, alimentícios, combustíveis renováveis, entre outros (Sarquis et al., 2020; Silva et al., 2018). Tais oleaginosas apresentam características peculiares sendo amplamente utilizadas pela população local para o tratamento de inúmeras doenças, bem como, são fontes renováveis e bioativas.

A biodiversidade amazônica apresenta uma grande fonte de plantas ricas em compostos bioativos, cuja exploração racional contribui para o desenvolvimento sustentável da região. Neste contexto, diversas espécies vegetais tornaram-se alvo de inúmeras pesquisas nos últimos anos, entre estas destacam-se a Carapa guianenses (andiroba) e a Euterpe oleracea (açaí) (Cabral et al., 2013).

O óleo das sementes de andiroba, encontrado na maior parte da América tropical, contém altos níveis de triacilgliceróis insaturados, o que o torna atraente para a indústria cosmética. Uma quantidade significativa de limonóides também confere a esse óleo amazônico várias propriedades medicinais. Além disso, o óleo também é uma matéria-prima potencial para a produção de biodiesel, e seus diversos usos intensificaram sua exploração extrativa nos últimos anos (Cabral et al., 2013). Assim como, a utilização do óleo de açaí vem ganhando destaque nas indústrias farmacêutica, de cosméticos e de alimentos, em função de seu elevado teor de compostos antioxidantes e conteúdo lipídico benéficos à saúde.

Deste modo, o objetivo deste trabalho foi realizar um levantamento bibliográfico acerca dos processos de extração dos óleos de andiroba e de açaí, e suas principais formas de utilização industrial. Já que estes são de significativa importância na economia das populações tradicionais do estado do Pará e para as indústrias de produtos naturais, e estão intimamente ligados à manutenção da saúde dos mesmos. Além de representarem grande relevância ambiental e sociocultural à região Amazônica.

\section{Metodologia}

A pesquisa foi elaborada com base teórica-metodológica e apresenta caráter descritivo e qualitativo (Evêncio et al., 2019; Estrela, 2018), a fim de avaliar as informações disponíveis até o momento acerca dos processos de extração dos óleos de andiroba e de açaí, bem como suas inúmeras aplicações industriais. Esta pesquisa foi baseada na análise da literatura já publicada, utilizando artigos, livros, teses, dissertações, sites institucionais, entre outros, disponibilizados para consulta nas bases de busca ScienceDirect, SciELO, Scopus, Web of Science, ACS (American Chemical Society), etc. Realizada no período 
de junho a agosto de 2021, utilizando-se as seguintes expressões e/ou palavras-chave, além de cruzamentos entre elas: andiroba, açaí, óleos vegetais, processos de extração, usos industriais (cosméticos, farmacêuticos, biocombustíveis, alimentício, etc), entre outros.

\section{3. Óleos Vegetais}

Os óleos vegetais são produtos naturais com vários componentes bioativos, formados principalmente de triglicerídeos (95-98\% em peso), e são extraídos de produtos que podem ser obtidos na floresta, como sementes, cascas, raízes, folhas, resinas ou frutos (Sharmin et al., 2015; Medeiros et al., 2018). Os triglicerídeos são compostos por três cadeias de ácidos graxos esterificados em uma única molécula de glicerol. Mono e diacilglicerol, fosfolipídios, tocoferóis, esteróis, carotenoides, polifenóis e água estão presentes em quantidades menores nos óleos vegetais (Yara-varón et al., 2017). A região amazônica é uma importante fonte de plantas que produzem diferentes óleos vegetais, e estes são usados pela população local para o tratamento de doenças.

\subsection{Andiroba}

A andiroba, Carapa guianensis Aublet., é uma arvore pertencente à família Meliaceae, amplamente utilizada na medicina tradicional brasileira e de áreas vizinhas à floresta amazônica. O nome andiroba (ãndi'roba) é de origem tupi, e significa óleo amargo (Nascimento et al., 2019; Henriques \& Penido, 2014). A árvore de andiroba é uma das mais importantes plantas medicinais utilizadas como fitoterápicos pelos povos indígenas e habitantes tradicionais da floresta amazônica.

A andirobeira é amplamente distribuída por toda a floresta amazônica, crescendo principalmente em regiões de várzea e áreas alagáveis ao longo dos igapós, podendo ser encontrada também em áreas de terra firme, incluindo Colômbia, Venezuela, Suriname, Guiana Francesa, Brasil, Peru, além do Paraguai e ilhas do Caribe (Kenfack, 2011). Caracteriza-se como uma árvore de grande porte que pode chegar até $50 \mathrm{~m}$ de altura, mas normalmente são encontrados espécimes entre 25-35 m; apresenta casca espessa, de cor avermelhada ou acinzentada que se descasca em grandes placas. A árvore produz uma noz marrom (ouriço arredondado) de quatro valvas, com cerca de 3-5 cm de diâmetro. $\mathrm{O}$ fruto, quando maduro, cai e libera suas sementes poligonais, ricas em um óleo amarelo-claro que pode ser extraído artesanalmente, por auxílio de equipamentos ou até mesmo com utilização de produtos químicos (Forget \& Kenfack, 2008; Nascimento et al., 2019).

Tradicionalmente, todas as partes da árvore são utilizadas pelos povos amazônicos, sendo o óleo, extraído das sementes, o mais utilizado na medicina popular, para tratar inflamações cutâneas, tumores e distensões musculares. Também é utilizado pelos indígenas em mistura com corante de urucum (Bixa orellana L.), como repelente contra insetos e como medicamento para parasitas dos pés (Sousa et al., 2019).

Das folhas e cascas é feito uma infusão, utilizada para amenizar a febre, tratar problemas intestinais, vermes e reumatismo. O extrato da casca também tem sido aplicado topicamente para tratar úlceras e outros problemas de pele, incluindo a psoríase (Forget \& Kenfack, 2008; Kenfack, 2011; Henriques \& Penido, 2014).

O sabor amargo da andiroba, o qual está presente em todas as suas partes é devido à presença de grupos químicos denominados limonóides, tetranortriterpenóides ou meliacinas, tais compostos são responsáveis pelos efeitos inseticidas, sendo produzidos pelas plantas como mecanismo de defesa contra insetos fitófagos (Senhorini et al., 2012).

A alta concentração desses componentes principalmente nas sementes pode explicar, pelo menos em parte, as propriedades terapêuticas desta espécie vegetal e o grande interesse pelas inúmeras pesquisas científicas. Os limonóides têm atraído muita atenção devido às suas elevadas atividades biológicas, incluindo atividades antifúngicas, bactericidas, antivirais e propriedades reguladoras do crescimento. (Henrique \& Penido, 2014). Os principais ácidos graxos encontrados no óleo de 
andiroba são o oléico, palmítico, esteárico, linoléico e palmitoleíco, com concentrações de 56,1\%,26,4\%, 7,6\%, 7,3\% e 0,4\%, respectivamente (Gonzales et al., 2008).

O óleo de andiroba serve de insumo para as indústrias cosméticas, farmacêuticas, alimentícias e têxteis, fazendo parte da composição de perfumes, produtos de higiene pessoal e de beleza, corantes e alimentos funcionais (Sarquis et al., 2020). O aumento da procura pelo óleo da andiroba no mercado de cosméticos se deu após o patenteamento de um creme hidratante e um creme anticelulite à base desse óleo pela empresa francesa Yves Rocher (Morais \& Gutjahr, 2012).

Essas finalidades atribuídas têm impulsionado o plantio de C. guianensis em consórcio com outras espécies ou na forma de monocultura, no Estado do Pará (Santos et al., 2014). Bem como a comercialização das sementes e do óleo de andiroba movimenta os mercados regionais e é fonte de renda de inúmeras famílias que se dedicam exclusivamente a coleta desta oleaginosa. O processo de beneficiamento do óleo agrega valor ao produto final além de potencializar suas qualidades (Sousa, et al., 2019). A escolha do processo de extração afeta diretamente a qualidade e quantidade do produto a ser extraído. O óleo é um dos remédios naturais mais vendidos na região amazônica, disponíveis em feiras, farmácias e por vendedores autônomos. Além de já está sendo comercializado para outras regiões do Brasil e exportado para outros países.

\subsection{Açaí}

O açaizeiro, Euterpe oleracea Mart., é uma palmeira pertencente à família Arecaceae, medindo de 3 a $20 \mathrm{~m}$ de altura e com caule liso de 7 a $18 \mathrm{~cm}$ de diâmetro. Frutifica durante todo o ano, com pico de produção nos meses de julho a dezembro, crescendo em áreas alagadas e de terras úmidas, com alta regeneração natural. Os frutos, chamados açaí, são globosos lisos, medindo $1,2 \times 1,3 \mathrm{~cm}$ de diâmetro, de coloração violeta na maturidade. Estes fornecem um óleo verde-escuro bastante utilizado na medicina popular (Oliveira, 2002; Bichara \& Rogez, 2011).

O Brasil é o principal produtor, consumidor e exportador de açaí, correspondendo a $42 \%$ da dieta da comunidade amazônica. O estado do Pará é o maior produtor e consumidor do fruto, respondendo por cerca de $95 \%$ da produção nacional. O fruto, na forma de polpa congelada, já vem sendo exportado para países como Estados Unidos, Japão, China e alguns países Europeus, sendo consumido nestes, preferencialmente como bebida energética (CONAB, 2019). Sendo de grande relevância socioeconômica para a região Amazônica, devido ao seu enorme potencial de aproveitamento integral do fruto. A principal e mais rentável forma de utilização, é a produção e comercialização da polpa de açaí, normalmente obtida por extração mecânica ou manual, e muito utilizada na alimentação popular e na produção industrial ou artesanal de sucos, sorvetes, picolés e na fabricação de geleias, licores, etc (Boeira et al., 2020). As folhas são utilizadas, tradicionalmente, para auxiliar na cobertura de casas, nas áreas de plantio dos açaizeiros, bem como para tratar picadas de cobra, dores musculares e no peito; as sementes, são utilizadas para artesanato e como adubo orgânico, e do caule extrai-se palmito (Yamaguchi et al., 2015). Outra aplicação da polpa de açaí é para produção de corante natural, uma vez que seus frutos maduros fornecem um pigmento da classe das antocianinas, cuja coloração pode variar de púrpura a verde-escuro azulado a depender do meio (ácido/básico) no qual o extrato foi preparado (Oliveira et al., 2002). Ainda pode-se obter um óleo a partir da polpa do fruto, que se caracteriza como um fluido viscoso de coloração verde escura, e distinto aroma remanescente de açaí (Pacheco-Palencia, Talcott, \& MertensTalcott, 2008).

O açaí é considerado um super alimento, pois sozinho contêm propriedades de uma dieta saudável, com sua combinação lipídica benéfica à saúde (50\% em base seca), teores relevantes de proteínas, fibras alimentares e compostos antioxidantes, além de apresentar baixo teor de açúcares (Rogez, 2000). Possui grande quantidade de antocianinas e compostos fenólicos benéficos à saúde, atuando na prevenção e combate de doenças cardiovasculares, neurodegenerativas, convulsões, e até leishmaniose, o que justifica constantes pesquisas acerca do tema (Yamaguchi et al., 2015). O açaí também possui uma grande quantidade de vitaminas (principalmente A, C e E) e minerais, especialmente Ferro, Zinco, Cobre, Potássio, e outros 
compostos, como esteróis e carotenoides (Bichara \& Rogez, 2011). Apresentando ação antioxidante, anti-inflamatória, antimicrobiana e anticarcinogênica (Ferreira, Rogez \& Herman, 2018).

O óleo presente nos frutos do açaizeiro representa aproximadamente $50 \%$ da matéria seca total da polpa e apresenta um perfil lipídico rico em ácidos graxos mono e poli-insaturados, característica que o assemelha ao azeite de oliva e de abacate, qualificando-o como um óleo comestível especial (Marques et al., 2016; Xiong et al., 2020), além de apresentar antocianinas e fitoesteróis. A composição de ácidos graxos do óleo do açaí é de 73,9\% de ácidos graxos insaturados, dentre estes predomina o ácido oléico (56,2\%), seguido do ácido linoléico (11,5\%) e linolênico (0,8\%), os ácidos graxos saturados principais são palmítico $(24,1 \%)$ e o esteárico $(1,6 \%)$, totalizando cerca de $27,5 \%$ de ácidos graxos saturados (PachecoPalencia, Duncan \& Talcott, 2009). Dentre os fitoesteróis presentes no óleo destacam-se o beta-sitosterol, o stigmasterol e o campesterol, utilizados pela indústria cosmética para prevenir o envelhecimento precoce, por promover o metabolismo celular e reduzir a inflamação (Morais \& Gutjahr, 2012).

Tal óleo se apresenta como um subproduto valioso dadas as suas propriedades sensoriais únicas e os seus potenciais benefícios à saúde, em especial sua elevada atividade antioxidante, em função do seu conteúdo fenólico, sendo os antioxidantes mais abundantes o $\alpha$-tocoferol - vitamina E (67\%) e os tocotrienóis, os quais previnem a oxidação lipídica do óleo, proporcionando estabilidade de armazenamento (Silva \& Rogez, 2013; Xiong, et al., 2020). O óleo ainda é rico em antioxidantes naturais apolares, entre estes, os principais detectados até agora foram os ácidos vanílico, síngico, protocatecuico, cafeíco, ferúlico, dímeros e trímeros de procianidina (Marques et al., 2016).

Devido aos seus vários benefícios à saúde, o açaí amazônico tem sido muito procurado pelas indústrias nacionais e internacionais dos setores alimentício, cosmético e farmacêutico (Pacheco-Palencia, Mertens-Talcott \& Talcott, 2008), sendo uma promissora alternativa aos óleos tradicionais.

A coloração verde-escura do óleo de açaí é devido à presença do pigmento clorofila, que é responsável por proporcionar efeitos benéficos à saúde. No entanto, é de extrema importância que os métodos de extração utilizados preservem tal pigmento, onde a temperatura está diretamente ligada à sua manutenção (Ferreira, Rogez \& Herman, 2018).

Como observado, o estudo e a valorização do óleo da polpa de açaí é recente, ganhando destaque deste os anos de 2010 e, até o momento, a maioria dos trabalhos relacionados a esse óleo se concentra nas aplicações biológicas e não nos processos de extração, que são úteis aos fabricantes. Até onde sabe-se, não existe tecnologia com otimização técnicoeconômica para a extração do óleo da polpa do fruto açaí, contando com os métodos físico-químicos convencionais utilizados em escala comercial (Yamaguchi et al., 2015). Esses métodos geralmente empregam solventes orgânicos, principalmente nhexano, prensa hidráulica e $\mathrm{CO}_{2}$ supercrítico (Batista et al., 2016). Embora proporcionem um bom rendimento de extração, são caros e de difícil manipulação em escala industrial.

\section{Métodos de Extração}

Os processos de extração de óleos vegetais são classificados como operações unitárias que visam à separação de determinados compostos, a partir de processos químicos, físicos ou mecânicos. As características dos óleos vegetais podem ser alteradas em virtude do método extração selecionado e das condições as quais ele é submetido, tais como altas temperaturas, secagem das sementes, irradiação, alta concentração de oxigênio, etc (Ribeiro et al., 2012).

Os métodos de extração de óleos vegetais comumente utilizados são físicos ou químicos, mediante a utilização de prensas, solventes orgânicos, $\mathrm{CO}_{2}$ supercrítico, etc., apesar de possuírem alto rendimento, são de difícil manipulação, apresentam custos relativamente elevados e no caso dos solventes ainda acarretam impactos negativos ao meio ambiente (Li et al., 2014). 
O método de extração deve visar o aproveitamento máximo do conteúdo lipídico da oleaginosa, buscando as melhores condições de processamento, promovendo desta forma um maior rendimento, redução de custos e aumento de produtividade. Os processos de extração de óleos e gorduras mais utilizados são a prensagem direta (ou extração mecânica), extração com solvente e extração com solvente associada à prensagem, além da extração por fluído supercrítico (Milhomen-Paixão et al., 2017; Santos, 2014). Os quais serão apresentados a seguir.

\subsection{Método artesanal ou tradicional}

\subsubsection{Andiroba}

O processo artesanal ou tradicional de extração do óleo das sementes de andiroba é longo, sendo este método adotado pelas comunidades ribeirinhas da região amazônica, embora com inúmeras variações nas etapas do processo, este pode ser divido em coleta e seleção das sementes, preparo da massa (cozimento das sementes até amolecimento / 45-60 minutos), secagem (fermentação por até 15 dias), extração (amêndoas cozidas amassadas manualmente), descanso para surgimento do óleo, decantação do óleo em bicas de alumínio, inox, etc, envase em frascos de vidro escuros e comercialização do óleo (Coelho et al., 2018; Brito et al., 2020). Este processo pode ser realizado à sombra ou ao sol, quando extraído à sombra, o óleo é considerado de melhor qualidade, já que apresenta menor índice de acidez, ou seja, oxidação, porém o processo é mais demorado (até 30 dias) (Coelho et al., 2018).

O azeite obtido no início do gotejamento é chamado de "virgem", apresenta cor esverdeada e as propriedades medicinais são mais acentuadas (Brito et al., 2020; Melo, Almeida \& Dantas, 2011), no entanto, o óleo amarelo-claro é de maior interesse comercial, pois apresenta maior estabilidade. Neste método, o manejo das sementes influencia diretamente no rendimento e qualidade do óleo, já que não há padronização, diferenciando consideravelmente em função do local de coleta e extração, bem como das condições climáticas (Pinto, Lira-Guedes \& Guimarães, 2019).

\subsubsection{Açaí}

O processamento manual (tradicional) do açaí ainda é realizada no interior da Região Norte, em especial no Estado do Pará, por populações que plantam açaí apenas para consumo próprio. Este método consiste em colheita, seleção dos frutos, lavagem por três vezes com água potável, imersão em água morna $\left(40^{\circ} \mathrm{C}\right)$ por 30 minutos (amolecimento da polpa), em seguida a água do molho é descartada e os frutos são colocados sobre uma peneira e macerados manualmente adicionando-se água potável para facilitar a extração da polpa de açaí, em seguida esta é novamente aquecida para separação do óleo sobrenadante. Essa exposição a temperaturas elevadas interfere na qualidade final do óleo, além de ser um método mais demorado e de baixo rendimento (Ferreira, Rogez \& Herman, 2018). Em contraposição, extrações químicas resultam em maior rendimento, contudo podem deixar resíduos no produto (Castro et al., 2021).

\subsection{Prensagem a frio ou extração mecânica}

Na extração mecânica ou prensagem há uma boa relação custo-benefício, uma vez que não requer nenhum tipo de composto químico, além de não gerar nenhum resíduo tóxico ao meio ambiente. No entanto, este método apresenta baixo rendimento, sendo recomendado para sementes oleaginosas, tal como a andiroba, amendoim (Arachis hypogaea L.), oliva (Olea europaea), babaçu (Orbignya phalerata), castanha do Pará (Bertholletia excelsa), girassol (Helianthus annuus), entre outras (Boas et al., 2012).

Dois tipos de prensa podem ser utilizados na extração de óleos vegetais, as prensas hidráulica e de rosca (mecânica). A eficiência destas, quando de baixa capacidade, podem ser aumentadas a partir da otimização das principais variáveis 
envolvidas no processo, como a pressão aplicada sobre a massa das amêndoas, prévio preparo da matéria-prima, temperatura de prensagem e teor de umidade das sementes (Mendonça et al., 2020).

Os equipamentos utilizados neste método vão desde os artesanais até os equipamentos industriais como as prensas contínuas, tipo "expeller", e as prensas hidráulicas. As prensas do tipo contínua apresentam maior capacidade, menor mão-deobra e menor investimento quando comparado com extração por solvente ou por fluido supercrítico. Já as hidráulicas, são utilizadas em instalações menores e condições de processamento mais amenas, como por exemplo a temperatura (Akoh \& Min, 2007; Mendonça et al., 2020).

Pesquisas realizadas por Ferreira et al., (2011), indicam que o método de extração por prensagem a frio produziu óleo de andiroba com elevado teor de compostos fenólicos e alta capacidade antioxidante, comparado ao método de extração por solvente, justificado pela baixa temperatura de extração. Já Mendonça e colaboradores (2020), desenvolveram uma pesquisa acerca dos rendimentos do óleo de andiroba extraído por prensa hidráulica a frio, controlando-se a umidade e temperatura de secagem das amêndoas. Neste trabalho verificou-se que o rendimento de extração do óleo foi melhor quando as sementes foram secas a $40^{\circ}$ e com umidade de $10 \%$. No entanto, estudos acerca dos diversos processos de extração do óleo de andiroba ainda são incipientes, havendo a necessidade de mais pesquisas e incentivos para padronização dos mesmos.

No caso da extração mecânica (prensagem a frio) do óleo de açaí utilizam-se máquinas despolpadoras elétricas de inox, onde previamente, os frutos são selecionados, limpos e imersos em água a $45^{\circ} \mathrm{C}$ por 60 minutos, em seguida são colocados na despolpadora com adição de água potável durante o processamento, o que facilita, sobremaneira, as operações de despolpamento e filtração. Em seguida a polpa deve ser desidratada, seca em estufa até peso constante à $45^{\circ} \mathrm{C}$ por 48 horas, esta passa para uma prensa hidráulica manual e o óleo é obtido e coletado, preferencialmente em vidro âmbar (Santos et al., 2013).

Para a obtenção de maiores rendimentos faz-se necessário o uso de frutos da mesma colheita, com curto período de armazenamento, bem como temperaturas superiores a $50^{\circ} \mathrm{C}$ aumentam o índice de peróxido do óleo, ocasionando perdas dos compostos bioativos, como as antocianinas (Xiong et al., 2020). Este é método é mais comumente utilizado pelas indústrias para produção de óleo de açaí extra virgem, uma vez que os produtos obtidos são de maior pureza e qualidade, preservando suas propriedades naturais.

\subsection{Extração com solventes}

Este método consiste na utilização de solventes orgânicos, como o hexano ou éter de petróleo para extração de óleo em sementes oleaginosas, polpa in natura de frutos, bem como no resíduo (torta) gerado após extração mecânica dos mesmos (AOAC, 2000). Quando se realiza a extração diretamente das sementes, estas devem ser previamente trituradas para aumentar a superfície de contato, e o solvente pode ser recuperado por destilação. Quando se utiliza a polpa de açaí, esta pode ser congelada a $-30^{\circ} \mathrm{C}$ e posteriormente liofilizada. Após extração, o óleo deve ser filtrado para remoção de possíveis partículas sólidas (Silva \& Rogez, 2013).

Estudos revelam que este método reduz o teor de ácidos graxos polinsaturados no óleo obtido da polpa de açaí em torno de $2,5 \%$ em relação ao extraído com enzimas, devido ao aumento da oxidação lipídica na presença de oxigênio (Nascimento et al., 2008). Este método ainda eleva os custos de produção e é mais prejudicial ao meio ambiente.

Recentemente, Castro e colaboradores (2021), realizaram um estudo para determinar as propriedades físico-químicas do óleo de açaí obtido por extração por solvente orgânico hexano, utilizando aparelho do tipo Soxhlet, obtendo baixos rendimentos, em virtude de utilizarem frutos inteiros. O teor lipídico obtido foi de 3,5\%, considerado inferior aos resultados encontrados por Buratto et al., (2021), que foi de 43,1\%, onde o óleo de açaí foi obtido a partir da polpa do fruto por extração pressurizada, evidenciando que o método de extração influencia diretamente no rendimento e qualidade do produto. 
Uma técnica alternativa ao método convencional de extração por solventes é a extração de óleo de andiroba por nbutano pressurizado, onde há controle de temperatura e pressão, reduzindo o tempo de extração e produzindo óleo de melhor qualidade, pois a extração desse óleo por hexano pode gerar algum resíduo no extrato, que pode ocasionar toxicidade (Novello et al., 2015).

\subsection{Extração por fluído supercrítico}

Este processo consiste na utilização de fluído supercrítico, onde o dióxido de carbono $\left(\mathrm{CO}_{2}\right)$ é o mais amplamente utilizado, por ser atóxico, não inflamável, não corrosivo e de fácil manipulação, permitindo operação supercrítica em baixas pressões e temperaturas próximas a ambiente (Ahmad et al, 2019). Esta técnica é muito utilizada nas indústrias farmacêuticas, alimentícias, químicas e de cosméticos devido ao grande interesse nesta tecnologia estar diretamente relacionado com as restrições ao uso de solventes orgânicos, além da redução no tempo de extração (Silva et al., 2016).

O processo de extração com fluidos supercríticos é considerado uma tecnologia de alta capacidade produtiva, apresentando diversas vantagens em relação aos métodos convencionais de extração de óleos vegetais, especialmente por ser uma tecnologia limpa e seletiva, levando a obtenção de extratos com composição química diferenciada (Sharif et al., 2014; Ahmad et al., 2019). É um processo que ocorre em duas etapas, a extração e separação do extrato do solvente.

Estudos relatam que o perfil de ácidos graxos obtidos por esta técnica apresenta alto rendimento e seletividade quando comparados a métodos tradicionais de extração (Arnáiz et al., 2012). Bem como, apresenta alta eficiência na extração e preservação de tocoferois (Nyam et al., 2010).

Neste método, alguns parâmetros devem ser controlados, tais como pré-tratamento da amostra, granulometria do material, temperatura, pressão, tempo, fluxo de solvente, entre outros, de modo a maximizar o rendimento dos compostos de interesse e minimizar a extração simultânea de compostos indesejáveis (Silva et al., 2016). Estes parâmetros influenciam na eficiência da extração. Em um estudo relatado por Batista et al. (2016), o óleo de açaí foi obtido a partir da polpa liofilizada do fruto, por extração com $\mathrm{CO}_{2}$ supercrítico, variando-se a densidade $\left(700,800\right.$ e $\left.900 \mathrm{Kg} / \mathrm{m}^{3}\right)$, temperatura $\left(50,60\right.$ e $\left.70^{\circ} \mathrm{C}\right)$ e pressão $(150,190,220,270,320,350,420$ e 490 bar). Sendo verificado que os melhores resultados foram obtidos nas condições experimentais de $70^{\circ} \mathrm{C} / 490$ bar.

Apesar do $\mathrm{CO}_{2}$ ser o solvente mais relatado na extração supercrítica, há a necessidade de se buscar novos solventes, com objetivo de otimizar e popularizar a técnica. A importância dos óleos vegetais para os diversos fins gera a necessidade de técnicas de extração com elevados rendimentos.

\subsection{Extração enzimática em meio aquoso}

Este método consiste na utilização de enzimas com atividades específicas, que hidrolisam a parede celular dos vegetais liberando o óleo em meio aquoso, que é separado da água por centrifugação. Esta técnica tem despontado como uma alternativa promissora para extração de óleos vegetais a partir de polpas de frutas, como o óleo de açaí, uma vez que não utiliza solventes orgânicos, se caracterizando como uma tecnologia limpa, sustentável, que minimiza os impactos ao meio ambiente, apesar de ser uma tecnologia de custo mais elevado que as demais (Nascimento et al., 2008; Ferreira, Rogez \& Herman, 2018).

Estudos sugerem que processos que utilizam a combinação de duas ou mais enzimas aumentam os rendimentos de extração do óleo de açaí em até $90 \%$ quando comparado com a utilização de uma única enzima, que aumenta o rendimento em cerca de 30 \% (Ferreira, Rogez \& Herman, 2018), facilitando a extração de óleo a nível industrial.

Em comparação aos processos convencionais de extração, o uso de meio aquoso é muito mais seguro e ecologicamente correto, bem como, o processo permite a extração simultânea do óleo e seus compostos bioativos, como por exemplo, vitaminas, compostos fenólicos, carotenoides, etc. No entanto, as principais limitações para a aplicação do processo, 
em escala industrial são o custo, tempo e disponibilidade das enzimas (Yusoff et al., 2015). Mesmo assim, a extração aquosa de óleos vegetais com auxílio de enzimas vem sendo amplamente utilizada, em nível industrial, devido as inúmeras vantagens em relação aos processos tradicionais, bem como produzir óleos de excelente qualidade.

\section{Usos Industriais}

\subsection{Industrias cosméticas e farmacêuticas}

Os óleos vegetais, em especial o de andiroba e o de açaí são amplamente utilizados como componentes básicos ou composto ativo de formulações cosméticas e farmacêuticas, uma vez que apresentam baixa toxicidade e são biocompatíveis com a pele. Há uma tendência mundial em substituir produtos cosméticos sintéticos pelos naturais e, assim atribuir à biotecnologia um papel cada vez mais relevante no aumento da competitividade tecnológica e industrial (Meneguetti et al., 2019; Carvalho, 2013). O mercado de cosméticos é um dos que mais cresce no mundo, devido a sua grande potencialidade em ativos que geram inúmeros produtos que agradam o mercado consumidor.

O óleo de açaí é um dos mais raros e valorizados da flora amazônica. Com sua altíssima concentração de diferentes bioativos antioxidantes (até 30 vezes mais do que o óleo de sementes de uva) é extremamente eficaz no combate à radicais livres na pele e previne o envelhecimento precoce. Este óleo apresenta-se como um novo ativo cosmético, originário da floresta Amazônica, que oferece inúmeros benefícios para a manutenção do equilíbrio cutâneo (Yara-Varon et al., 2017).

Várias empresas regionais, nacionais e internacionais vêm se destacando nas últimas décadas na produção de cosméticos e produtos farmacêuticos a base de óleos naturais amazônicos, entre estes os óleos de andiroba e de açaí. A seguir, algumas destas empresas foram destacadas.

A Indústria Amazonoil LTDA, localizada na cidade de Belém - Pará, processa todos os óleos e manteigas de espécies amazônicas na própria sede, entre estas matérias-primas estão o açaí e a andiroba. Estas são prensadas a frio, secas até $60^{\circ} \mathrm{C}$, sem adição de conservantes ou qualquer outro aditivo químico. Tal indústria valoriza o extrativismo sustentável da floresta amazônica, fortalecendo a economia local e preservando o meio ambiente. Que comercializam produtos para pele e cabelo, por exemplo, os quais possuem entre outros ativos amazônicos, óleos de açaí e andiroba (Amazon Oil, 2021).

Outra empresa que se destaca no cenário amazônico é a Beraca LTDA, fornecedora líder de ingredientes naturais extraídos da floresta Amazônica e outros biomas brasileiros. Esta produz, entre outros, óleos de açaí e andiroba com certificação orgânica. Os óleos estão disponíveis nas versões grau cosmético, não refinado e refinado - óleos refinados seguem padrões de alta qualidade para melhor desempenho em formulações cosméticas. A empresa produz ativos de alta performance, o que agrega valor a um grande número de marcas que operam em todo o mundo. O extrato de açaí possui elevadas concentrações de antocianinas. O açaí produzido na empresa é zero resíduo, com aproveitamento integral da espécie, onde todas as suas partes são aplicadas em diferentes ingredientes cosméticos, como extrato, óleo e esfoliante (BERACA, 2021).

Ainda se destaca a Natura Cosméticos, uma multinacional brasileira, que a partir do extrativismo vegetal com cooperativas do entorno da Região metropolitana de Belém, Capital do Pará, obtém as matérias-primas para a extração dos óleos amazônicos, muito utilizados na produção de cosméticos e biocosméticos. As comunidades fornecedoras, parceiros desta empresa, aplicam técnicas de sustentabilidade para extrair os óleos das plantas que constituem a biodiversidade da região, entre estas matérias-primas estão o açaí, murumuru, andiroba, buriti, entre outros. Os quais produzem uma linha especifica de cosméticos, que utilizam os óleos de açaí ou de andiroba, movimentando os mercados nacionais e internacionais, sendo produzidos sabonetes, hidratantes, óleos corporais, produtos para cabelo, entre outros. A empresa conta com uma extensa rede de cooperação que envolve as comunidades extrativistas, universidades, ONG'S, empresas e órgãos governamentais (Natura Cosméticos S/A, 2021). 
Há outras indústrias de pequeno e médio porte na região metropolitana de Belém - Pará, bem como em pequenas cidades no interior do estado que comercializam produtos cosméticos a base dos óleos de andiroba e/ou óleo de açaí, como por exemplo, sabonetes naturais produzidos artesanalmente. Além disso, esses óleos são muito comercializados puros, sendo vendidos em feiras livres, por vendedores autônomos, podem ser encontrados ainda em vários sites da internet. A empresa francesa ROCHER YVES BIOLOG VEGETALE detém uma patente referente a uma composição cosmética ou farmacêutica contendo extrato de Andiroba (Morais \& Gutjahr, 2012).

Também são encontradas velas produzidas com óleo de andiroba, muito utilizadas, na medicina popular, como repelente, não tópico, eficaz no combate ao mosquito Aedes aegypti, vetor da febre amarela e da dengue. Tais produtos são produzidos por pequenas empresas do Estado do Pará e abastecem o mercado local, já sendo comercializadas para outros estados do Brasil, mesmo não havendo comprovação de eficácia nem aprovação pela Anvisa, até o momento (Fontenelles \& Yamaguchi, 2018).

O óleo de andiroba também vem sendo utilizado como biorepelente, juntamente com outros óleos amazônicos, como copaíba e citronela. Este não agride o meio ambiente e ajuda a prevenir contra doenças como a dengue, Chikungunya, zica, malária e febre amarela (Maia, 2017). Este é produzido por pequenas empresas na Região do baixo Amazonas, no Pará. Segundo estudos de Prophiro et al., 2012, o efeito larvicida residual do óleo de andiroba, em 72 h apresentou 100\% de mortalidade para o vetor do Aedes aegypti. Com base nisso diversos estudos vêm sendo desenvolvidos para o desenvolvimento de produtos à base desse óleo, tais como as microcápsulas de andiroba. Estas permitem a utilização do óleo de andiroba como inseticida na forma sólida para liberação controlada a fim de prolongar seu efeito inseticida. Tal efeito é característico da presença de compostos do tipo terpenos, limonóides ou meliacinas (Senhorini et al., 2016).

Ultimamente, foi comprovado pela primeira vez a atividade larvicida do ácido graxo livre de óleo de andiroba, inserido em uma matriz biopolimérica de fibroína de seda, um polímero natural encontrado em glândulas de artrópodes como o bicho-da-seda, este aumenta a biodisponibilidade e biodistribuição do ativo em meio aquoso, sendo consideradas promissoras alternativas aos larvicidas sintéticos contra os vetores do Aedes aegypti que são a base de piretróides e que causam sérios danos ambientais e à saúde (Sarquis et al., 2020).

Recentemente, o óleo de açaí foi utilizado na formulação de bebidas fermentadas probióticas, a qual possui boas características nutricionais, principalmente na constituição de macronutrientes e compostos fenólicos, se caracterizando como um alimento funcional (Guimarães et al., 2020). O óleo de açaí também é muito utilizado na culinária.

\subsection{Outras utilizações industriais}

\subsubsection{Produção de polímeros}

Inúmeras pesquisas vêm sendo realizadas com base na utilização dos óleos de andiroba e açaí para outras finalidades diferentes do ramo de cosméticos ou farmacêuticos.

O uso de óleos vegetais, tal como o óleo de andiroba, como polios vêm despontando nos últimos anos, em virtude de seus custos relativamente baixos e uma boa disponibilidade (Pillai et al., 2016). Recentemente foi relato o uso de polios de óleo de andiroba para produção de espumas flexíveis de poliuretano utilizando catálise enzimática (Silva, Cardoso \& Petzhold, 2018). Os óleos vegetais, para serem utilizados como polios devem ser modificados quimicamente através da introdução de grupos hidroxila $(\mathrm{OH})$, transesterificação via glicerólise alcalina para produção de monoacilglicerol (Echeverri et al., 2011), ou por catálise enzimática. Esta é uma alternativa promissora para contornar os baixos rendimentos e custos mais elevados dos métodos convencionais para produção de polios. Na catálise enzimática do óleo de andiroba são utilizadas lipases, que atuam naturalmente na hidrolise do triacilglicerol. E em determinadas condições, como excesso de glicerol e uso de solventes orgânicos, é possível favorecer a formação de monoacilglicerol, processo este denominado de glicerólise enzimática (Solaesa 
et al., 2016). Este processo, em comparação com os convencionais, pode ser realizado sob condições de temperatura e pressão menores, o que auxiliam na preservação das propriedades do óleo vegetal (Luque \& Cervero, 2014).

A composição de ácidos graxos do óleo de andiroba, tais como os ácidos oleico, esteárico e palmítico, sugere que o óleo de andiroba possui grande potencial para a produção de polióis e como matéria-prima para a produção de materiais poliméricos, como as espumas flexíveis de poliuretano (Iha et al., 2014; Silva, Cardoso \& Petzhold, 2018). Estes materiais são polímeros versáteis, podendo ser utilizados industrialmente, como tintas e revestimentos, materiais de isolamento térmico e biomateriais (Liu et al., 2017).

\subsubsection{Produção de biocombustíveis}

Nos últimos anos, as crescentes preocupações com o meio ambiente e o desenvolvimento de processos industriais sustentáveis preconizam as pesquisas por fontes alternativas de geração de energia. Assim, a produção de biocombustíveis a partir de fontes renováveis têm se tornado uma promissora solução, já que reduz a emissão de poluentes (Ambat et al., 2018).

Estudos recentes relatam à utilização de óleos vegetais, incluindo o óleo de andiroba na produção de biocombustíveis, utilizando óxido de nióbio impregnado com sódio $\left(\mathrm{Na} / \mathrm{Nb}_{2} \mathrm{O}_{3}\right)$ como catalisador heterogêneo (Carvalho et al., 2020). Neste trabalho verificou-se alta atividade catalítica para todos os óleos testados, com rendimentos superiores a 95\%. Gerando biocombustíveis que atendem as especificidades dos padrões internacionais. Tais matérias primas são abundantes em locais carentes de fontes de energia, o que vêm estimulando pesquisas científicas, garantindo benefícios energéticos, econômicos e ambientais. O óleo de açaí também apresenta grande potencial para produção de biocombustível, em função da sua composição diversificada de ácidos graxos, entre estes o oleico apresenta maior concentração (60\%). Dabaja, Bizzo e Perreira (2018) desenvolveram uma pesquisa para produção de biodiesel a partir de óleo de açaí empregando lipase comercial imobilizada em suporte de baixo custo. A síntese de biodiesel, nas condições utilizadas, alcançou um rendimento de 51,5\% em $12 \mathrm{~h}$ de reação.

\section{Considerações Finais}

Os óleos de andiroba e de açaí apresentam enorme potencial de comercialização pelas indústrias regionais, nacionais e até internacionais nos ramos de cosméticos, farmacêuticos, alimentos e de energia, em função de suas propriedades terapêuticas únicas, como elevado poder antiinflamátorio, antimicrobiano, etc. E no caso do óleo de açaí ainda pode ser utilizado como alimento funcional.

Várias cooperativas, órgãos governamentais e universidades já elaboram diretrizes para à extração do óleo de andiroba pelas comunidades produtoras, uma vez que este é de grande importância sociocultural e econômica, já que utilizam este óleo como remédio natural. Agregando valor à matéria-prima e garantindo renda extra às populações que dependem da extração desse produto. Mas, apesar do grande potencial do óleo de andiroba, ainda são necessários mais pesquisas e investimentos para que o mesmo seja utilizado industrialmente.

Neste estudo foi observado que a valorização do óleo de açaí é relativamente recente, ganhando destaque na última década e, até o momento, a maioria dos trabalhos relacionados a esse óleo se concentra nas aplicações biológicas e não nos processos de extração, que são úteis aos fabricantes. Bem como, ainda não existe uma tecnologia com otimização técnicoeconômica para a extração do óleo.

Estes óleos podem ser extraídos por vários métodos, entre os quais podem ser citados o artesanal, extração mecânica (prensagem a frio), por fluído supercrítico, enzimas, solventes orgânicos (hexano), entre outros. No entanto, observa-se que estas ainda são utilizadas em caráter experimental, embora proporcionem um bom rendimento de extração, são caras e de 
difícil manipulação em escala industrial, principalmente as de extração por fluído supercrítico, a enzimática e o uso solventes químicos, este último ainda gera resíduos tóxicos. Sendo a técnica de extração por prensagem a frio dos óleos avaliados a mais utilizada industrialmente, por produzir compostos de boa qualidade e não utilizarem nenhum tipo de aditivo. Ainda há a necessidade de se explorar técnicas para o processamento industrial dos óleos de andiroba e açaí, visando maiores rendimentos e preservando seus compostos bioativos. Bem como, desenvolver novas pesquisas acerca dos demais métodos de extração apresentados, de modo a reduzir os custos operacionais, em especial o por extração supercrítica, uma vez que é uma tecnologia limpa e de alto rendimento.

Verificou-se também que a maior parte da produção dos óleos de andiroba e açaí ainda são comercializados por industrias locais, e destinados aos mercados de cosméticos e farmacêutico, havendo a necessidade de pesquisas e mais investimentos para que esses óleos sejam utilizados em outros setores industriais.

Deve-se levar em consideração também à conservação da biodiversidade local através do manejo sustentável da andiroba e do açaí, já que valorizar essas matérias-primas é garantia de preservação do meio ambiente, além de impulsionar a economia local.

Além disso, os dados discutidos nesta revisão destacam o interesse em pesquisas futuras para investigar mais detalhadamente a produção de biocombustíveis obtidos a partir de óleos vegetais amazônicos, que são fontes alternativas para a geração de energia renovável.

\section{Referências}

Ahmad, T.; Masoodi, F. A.; Rather, S. A.; Wani, S. M. \& Gull, A. (2019). Supercritical Fluid Extraction: A Review. Journal og Biological and Chemical Chronicles., 5 (1), 114-122. http://dx.doi.org/10.33980/jbcc.2019.v05i01.019.

Akoh, C. C. \& Min, D. B. (2007). Food lipids: chemistry, nutrition and biotechnology. New York: CRC Press.

Ambat, I.; Srivastava, V. \& Sillanpää, M. (2018). Recent advancement in biodiesel production methodologies using various feedstock: A review. Renewable Sustainable. Energy Reviste, 90, 356-369. https://doi.org/10.1016/j.rser.2018.03.069

Amazon Oil. (2021). Andiroba e Açaí. https://www.amazonoil.com.br/pt/produtos-da-floresta.

AOCS Methods, (2000). AOCS Bd 3-52 em Official Methods and Recommended Practices of the American Oil Chemists' Society; 5th ed.; Firestone, D., ed.; AOCS Press: Champaign.

Arnáiz, E.; Bernal, J.; Martín, M. T.; Nozal, M. J.; Bernal, J. L., \& Toribio, L. (2012). Supercritical fluid extraction of free amino acids from broccoli leaves. Journal of chromatography A, 49-53. https://doi.org/10.1016/j.chroma.2012.04.066.

Batista, C. C. R.; Oliveira, M. S.; Araújo, M. E.; Rodrigues, A. M. C.; Botelho, J. R. S.; Souza Filho, A. P. S.; Machado, N. T. \& Carvalho Junior, R. N. (2016). Supercritical $\mathrm{CO}_{2}$ extraction of açaí (Euterpe oleracea) berry oil: Global yield, fatty acids, allelopathic activities, and determination of phenolic and anthocyanins total compounds in the residual Pulp. Journal Supercritical Fluids, 107, 364-369. http://dx.doi.org/10.1016/j.supflu.2015.10.006.

Beraca Ingredientes Naturais S/A. (2021) Nossos produtos. https://www.beraca.com/nossos-produtos/

Bichara, C. M. G. \& Rogez, H. (2011). Chapter 1: Açaí (Euterpe oleracea Martius). In: Yahia, E. M. (Ed.), Postharvest Biology and Technology of Tropical and Subtropical Foods: Açai to Citrus, vol. 2. Woodhead Publishing, Oxford, England, p. 1-23.

Boeira, L. S.; Freitas, P. H. B.; Uchôa, N. R.; Bezerra, J. A.; Cád, S. V.; Duvoisin, S. J.; Albuquerque, P. M.; Mar, J. M.; Ramos, A. S.; Machado, M. B. \& Maciel, L. R. (2020). Chemical and sensorial characterization of a novel alcoholic beverage produced with native acai (Euterpe precatoria) from different regions of the Amazonas state. LWT, 117, 108632. https://doi.org/10.1016/j.1wt.2019.108632.

Brito, A. D.; Silva, T. F. A. Coelho, R. F. R., \& Rosal, L. F. (2020). Saberes e práticas tradicionais da extração do óleo de Carapa guianenses Abul. (andiroba) em área de várzea do município de Igarapé-Mirin, PA. Revista Brasileira de Agroecologia, 15 (3), 110-122. https://doi.org/10.33240/rba.v15i3.23165.

Buratto, R. T., Cocero, M. J., \& Martín, A. (2021) Characterization of industrial açaí pulp residues and valorization by microwave-assisted extraction. Chemical Engineering and Processing - Process Intensification, 160, 108269. https://doi.org/10.1016/j.cep.2020.108269.

Castro, R. C.; Costa, L. F. S.; Costa, L. M. D. A., \& Martins, G. B. C. (2021). Extração e análises físico-químicas do óleo de açaí (Euterpe oleracea Mart.). Research, Society and Development, 10 (8), 1-5. http://dx.doi.org/10.33448/rsd-v10i8.17358.

Cabral, E. C.; Cruz, G. F.; Simas, R. C.; Sandivo, G. B.; Gonçalves, L. V.; Leal, R. V. P.; Silva, R. C.; Silva, J. C. T.; Barata, L. E. S.; Cunha, V. S.; França, L. F.; Daroda, R. J.; Sá, G. F.; Eberlin, M. N. (2013). Typification and quality controlo f the Andiroba (Carapa guianensis) oil via mass spectrometry gingerprinting. Analytical Methods, 5, 1385-1391. https://doi.org/10.1039/c3ay25743f. 


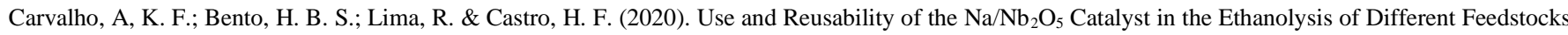
for Biofuel Production: Confirmation of Heterogeneity of the Catalyst. Energy Fuels, 34, 7105-7111. https://dx.doi.org/10.1021/acs.energyfuels.0c00246.

Carvalho, B. G. G. (2013). Avaliação preliminar de fitocosmético preparado com óleo de açaí. Monografia. Faculdade Educação e meio Ambiente.

Coelho, A. A.; Gama, J. R. V.; Ribeiro, R. B. S.; Oliveira, F. A. (2018). Andiroba: usos e extração do óleo em área de assentamento no oeste paraense. Terceira margem Amazônia, 3 (11), 1-16. https://doi.org/10.36882/2525-4812.2018v3i11p\%25p.

Conab - Companhia nacional de abastecimento (2019). Açaí (fruto) - Análises do mercado agropecuário e extrativo. https://www.conab.gov.br/infoagro/item/download.

Dabaja,M. Z.; Bizzo, B. M., \& Pereira, E. B. (2018). Síntese de biodiesel a partir do açaí empregando lipase comercial imobilizada em suporte de baixo custo. Revista da Universidade Vale do Rio Verde, 16 (2), 1-11. http://dx.doi.org/10.5892/ruvrd.v16i2.5016.

Echeverri, D. A.; Cardeño, F., \& Rios, L. A. (2011). Glycerolysis of soybean oil with crude glycerol containing residual alkaline catalysts from biodiesel production. JAOCS J. Am. Oil Chem. Soc. 88, 551-557. http://dx.doi.org/10.1007/s11746-010-1688-5.

Estrela, C. (2018). Metodologia Científica: Ciência, Ensino, Pesquisa - 2a ed., Editora Artes Médicas, 743p.

Evêncio, K. M. M.; Teixeira, S. L.; Rodrigues, K. G. F. C.; Feitosa, F. A., \& Fontes, W. J. S. (2019). Dos tipos de conhecimento às pesquisas qualitativas em educação. Id on line Revista Científica Multidisciplinar e de Psicologia, 13 (47), 440-452. https://doi.org/10.14295/idonline.v13i47.2105.

Ferreira, B. S.; Almeida, C. G.; Faza, L. P.; Almeida, A.; Diniz, C. G.; Silva, V. L.; Grazul, R. M., \& Hyaric, M. L. (2011). Comparative properties of Amazonian oils obtained by diferente extraction methods. Moleculles, 16, 5875-5881. https://doi.org/ 10.3390/ molecules16075875.

Ferreira, E. S.; Rogez, H. L. G., \& Herman, C. A. N. P. (2018). Effect of the combination of enzymatic preparations on the aqueous extraction yield of the oil from the pulp of euterpe oleracea fruit. Brazilian Journal of Chemical Engineering, 35 (4), 1193-1201. https://dx.doi.org/10.1590/0104$6632.20180354 \mathrm{~s} 20170305$.

Fontenelles, A. B. L., \& Yamaguchi, K. K. L. (2018). Uso de óleo de andiroba (Carapa guianensis) na produção de velas artesanais como instrumento para o ensino de Química. Exatas Online, 9 (2), 39-52. http://www2.uesb.br/exatasonline/images/V9N2pag39-52.pdf

Forget, P. M. \& Kenfack, D. (2008). Carapa.org. A website dedicated to trees in the genus Carapa (Meliaceae). Taxinomy, biology, ecology and uses. Muséum National d'Histoire Naturelle, Brunoy, France.

Freitas, S. P.; Silva, O. F.; Miranda, I. C., \& Coelho, M. A. Z. (2007). Extração e fracionamento simultâneo do óleo de castanha do Brasil com etanol. Ciencia e Tecnologia de Alimentos, Campinas, 27 (supl.), 14-17. https://doi.org/10.1590/S0101-20612007000500002.

Gonzales, W. A.; Machado, R. M.; Barreto, E. J. F.; Dall’Oglio, E. L.; Correia, J. C.; Borges, L. E. P.; Almeida, M. D.; Pastura, N. M. R.; Mendonça, N. B.; Sousa, P. T.; Nunes, P. P.; Rodrigo, R. M. S.; Souza, O. L., \& Rodrigues, V. (2008). Biodiesel e óleo vegetal in natura: soluções energéticas para a Amazônia. $1^{a}$ Edição - Brasília: Ministério de Minas e Energia. https://www.mme.gov.br/luzparatodos/downloads/Solucoes.pdf.

Guimarães, S. C. N.; Alves, D. T. V.; Souza, R. B. M.; Costa, C. E. F.; Melo, K. C.; Oliveira, I. S.; Soares, S. D., \& Santos, O. V. (2020). Desenvolvimento de formulações fermentadas probióticas mistas enriquecidas com óleos de frutos amazônicos. Brazilian Journal of Development, 6, (3), 10882-10901. http://doi.org/10.34117/bjdv6n3-093.

Henriques, M. G. \& Penido, C. (2014). The therapeutic properties of Carapa guianensis. Current Pharmaceutical Design, 20 (6), 850-856. https://dx.doi.org/10.2174/13816128113199990048.

Iha, O. K.; Alves, F. C. S. C.; Suarez, P. A. Z.; Silva, C. R. P.; Meneghetti, M. R., \& Meneghetti, S. M. P. (2014). Potential application of Terminalia catappa L. and Carapa guianensis oils for biofuel production: physical-chemical properties of neat vegetable oils, their methyl-esters and bio-oils (hydrocarbons). Industrial Crops and Products, 52, 95-98. http://dx.doi.org/10.1016/j.indcrop.2013.10.001.

Kenfack, D. A. (2011). A synoptic revision of Carapa (Meliaceae). Harvard Papers in Botany, 16 (2), 171-231. https://doi.org/10.3100/0.25.016.0201.

Kundu, J.; Chung, Y. I.; Kim, Y. H.; Tae, G. \& Kundu, S.C. (2010). Silk fibroin nanoparticles for cellular uptake and control release. International Journal of Pharmaceutics., 388, 242-250. https://doi.org/ 10.1016/ j.ijpharm.2009.12.052.

Li, Y.; Fine, F.; Tixier, A. S. F.; Vian, M. A.; Carre, P.; Pages, X. \& Chermat, F. (2014). Evaluation of alternative solventes for improvement of oil extraction from rapeseeds. Comptes Rendus Chimie, 17 (3), 242-251. https://doi.org/10.1016/j.crci.2013.09.002.

Liu, X.; Niu, Y.; Chen, K. C., \& Chen, S. (2017). Rapid hemostatic and mild polyurethane-urea foam wound dressing for promoting wound healing. Mater. Sci. Eng. C, 71, 289-297. http://dx.doi.org/10.1016/j.msec.2016.10.019.

Luque, S. \& Cervero, J. M. (2014). Novozym 435-catalyzed synthesis of fatty acid ethyl esters from soybean oil for biodiesel production. Biomass Bioenergy, 61, 131-137. http://dx.doi.org/10.1016/j.biombioe.2013.12.005.

Maia, A. C. (2017). Biorepelente com óleos essenciais de plantas da Amazônia é alternativa contra insetos https://redesfito.far.fiocruz.br/index.php/noticias/385-biorepelente-com-oleos-essenciais-de-plantas-da-amazonia-e-alternativa-contra-insetos.

Marques, E. S.; Froder, J. G.; Carvalho, J. C. T.; Rosa, P. C. P.; Perazzo, F. F. \& Maistro, E. L. (2016). Evaluation of the genotoxicity of Euterpe oleraceae Mart. (Arecaceae) fruit oil (açai), in mammalian cells in vivo. Food and Chemical Toxicology, 93, 13-19. https://dx.doi.org/ 10.1016/j.fct.2016.04.018

Medeiros, R. S.; Vieira, G.; Almeida, D. R. A. \& Tomazello, M. (2018). New information for managing Copaifera multijuga Hayne for oleoresin yield. Forest Ecology Management, 414, 85-98. https://doi.org/10.1016/j.foreco.2018.02.009. 
Melo, M. S.; Almeida, E. C. \& Dantas, J. B. (2011). Boas práticas de manejo e extração de óleo vegetal de andiroba. $1^{\text {a }}$ ed. Santarém: IBAMA/FFEM/ADF.

Mendonça, A. P.; Almeida, F. A. C.; Oliveira, A. S.; Rosa, J. C.; Araújo, M. E. R., \& Sampaio, P. T. B. (2020). Extração de óleo de andiroba por prensa: rendimento e qualidade de óleo de sementes submetidas a diferentes teores de água e temperatura de secagem. Scientia Florestalis. 48 (125), e2995. https://doi.org/10.18671/scifor.v48n125.09.

Meneguetti, N. F. P.; Meneguetti, D. U. O., \& Siviero, A. (2019). Biotechnological potential of the Carapa guianensis, Bertholletia excelsa and Copaifera spp. Oils. Journal of Medicinal Plants Research, 13 (17), 413-422. https://doi.org/ 10.5897/JMPR2019.6828.

Milhomem-Paixão, S. S. R.; Fascineli, M. L.; Muehlmann, L. A.; Melo, K. M.; Salgado, H. L. C.; Joanitti, G. A.; Pieczarka, J. C.; Azevedo, R. B.; Santos, A. S., \& Grisolia, C. K. (2017). Andiroba Oil (Carapa guianensis Aublet) Nanoemulsions: Development and Assessment of Cytotoxicity, Genotoxicity, and Hematotoxicity. Journal of Nanomaterials, 2017 (1), 1-11. https://doi.org/10.1155/2017/4362046.

Morais, L. R. B., \& Gutjahr, E. (2012). Química de oleaginosas: valorização da biodiversidade amazônica. Belém, PA: Ed. do autor.

Nascimento, R. J. S.; Couri, S.; Antoniassi, R. \& Freitas, S. P. (2008). Composição em ácidos graxos do óleo da polpa de açaí extraído com enzimas e com hexano. Revista Brasileira de Fruticultura, 30 (2), 498-502. https://dx.doi.org/10.1590/S0100-29452008000200040.

Nascimento, G. O.; Souza, D. P.; Santos, A. S.; Batista, J. F.; Rathinasabapathi, B.; Gagliardi, P. R., \& Gonçalves, J. F. C. (2019). Lipidomic profiles from seed oil of Carapa guianensis Aubl. And Carapa vasquezii Kenfack and implications for the control of phytopathogenic fungi. Industrial Crops and Products., 129, 67-73. https://doi.org/10.1016/j.indcrop.2018.11.069

Natura Cosméticos S/A. (2021). Biocosméticos. https://www.naturabrasil.fr/pt-pt/acerca-da-natura-brasil/as-nossas-gamas/ekos.

Novello, Z.; Scapinello, J.; Magrob, J. D.; Zinc, G.; Luccio, M. D.; Tres, M. V. \& Oliveira, J. V. (2015). Extraction, chemical characterization and antioxidant activity of andiroba seeds oil obtained from pressurized n-butane. Industrial Crops and Products, 76, 697-701. http://dx.doi.org/10.1016/j.indcrop.2015.07.075

Nyam, K. L.; Tan, C. P.; Karim, R.; Lai, O. M.; Long, K., \& Man, Y. B. C. (2010). Extraction of tocopherol-enriched oils from Kalahari melon and roselle seeds by supercritical fluid extraction (SFE-CO $)$. Food Chemistry, 119 (3), 1278-1283. https://doi.org/10.1016/j.foodchem.2009.08.007.

Oliveira, M. S. P.; Carvalho, J. E. U.; Nascimento, W. M. O. \& Müller, C. H. (2002). Cultivo do açaizeiro para produção de fruto. Ministério da agricultura, pecuária e abastecimento. BELÉM: EMBRAPA, Circular técnica n 26.

Pacheco-Palencia, L. A.; Talcott, S. T., \& Mertens-Talcott, S. (2008). Absorption and biological activity of phytochemical-rich extracts from açaí (Euterpe oleracea) pulp and oil in vitro. Journal of Agricultural and Food Chemistry, 56 (10), 3593-3600. https://doi.org/ 10.1021 / jf8001608.

Pacheco-Palencia, L. A.; Duncan, C. E. \& Talcott, S. T. (2009). Phytochemical composition and thermal stability of two commercial açai species, Euterpe oleracea and Euterpe precatória. Food Chemistry, 115, 1199-1205. https://doi.org/10.1016/j.foodchem.2009.01.034.

Pereira, C. M. S.; Assis, W. S. \& Araújo, C. S. (2015). O manejo da Andiroba e a contribuição para a preservação ambiental: o caso do Grupo de Trabalhadoras Artesanais e Extrativistas (GTAE) do Projeto de Assentamento Agroextrativista Praialta Piranheira (PAE)-PA. Cadernos de Agroecologia, 10 (3), 1-7. http://revistas.aba-agroecologia.org.br/index.php/cad/article/view/19234/13300.

Pillai, P. K. S.; Li, S.; Bouzidi, L., \& Narine, S. S. (2016). Metathesized palm oil: fractionation strategies for improving functional properties of lipid-based polyols and derived polyurethane foams. Industrial Crops \& Products, 84, 273-283. http://dx.doi.org/10.1016/j.indcrop.2016.02.021.

Pinto, E. R.; Lira-Guedes. A. C., \& Guimarães. (2019). Boas práticas para produção de óleo de andiroba. 32p. Tefé, AM: IDSM - Instituto de Desenvolvimento Sustentável Mamirauá. https://www.mamiraua.org.br/documentos/098c65b4178ed3236d4f2f88fdc046e4.pdf

Prophiro, J. S.; Silva, M. A. N.; Kanis, L. A.; Rocha, L. C. B. P.; Duque-Luna, J. E. \& Silva, O. S. (2012). First report on susceptibility of wild Aedes aegypti (Diptera: culicidae) using Carapa guianensis (Meliaceae) and Copaifera sp. (Leguminosae). Parasitology Research, 110, 699-710. https://doi.org/ 10.1007 / s00436-011-2545-7.

Ribeiro, M. C.; Vilas Boas, E. V. B; Riul, T. R.; Pantoja, L.; Marinho, H. A. \& Santos, A. S. (2012). Influence of the extraction method and storage time on the physicochemical properties and carotenoid levels of pequi (Caryocar brasiliense Camb.) oil. Ciência e Tecnologia de Alimentos, Campinas, 32 (2), 386392. https://dx.doi.org/10.1590/S0101-20612012005000053.

Rogez, H. (2000). Açaí: preparo, composição e melhoramento da conservação. Belém: EDUFA, 313p.

Santos, M. F. G.; Marmesat, S.; Brito, E. S.; Alves, R. E., \& Dobarganes, M. C. (2013). Major components in oils obtained from Amazonian palm fruits. Grasas y Aceites, 64 (3), 328-334. https://doi.org/10.3989/gya.023513.

Santos, M. N.; Cunha, H. F. A.; Lira-Guedes, A. C.; Gomes, S. C. P. \& Guedes, M. C. (2014). Saberes tradicionais em uma unidade de conservação localizada em ambiente periurbano de várzea: etnobiologia da andirobeira (Carapa guinensis Aublet). Boletim do Museu Paraense Emílio Goeld. Ciências Humanas, 9 (1), 93-108. https://10.1590/s1981-81222014000100007.

Santos, M. J. T. (2014). Aproveitamento de resíduos da indústria de óleos vegetais produzidos na Amazônia. Dissertação de mestrado. Universidade Federal do Pará. Instituto de Tecnologia. Programa de Pós-Graduação em Ciência e Tecnologia de Alimentos.

Sarquis, I. R.; Sarquis, R. S. F. R.; Marinho, V. H. S.; Neves, F. B.; Araújo, I. F.; Damasceno, L. F.; Ferreira, R. M. A.; Souto, R. N. P.; Carvalho, J. C.T. \& Ferreira, I. M. (2020). Carapa guianensis Aubl. (Meliaceae) oil associated with silk fibroin, as alternative to traditional surfactants, and active against larvae of the vector Aedes aegypti. Industrial Crops \& Products, 157, 112931. https://doi.org/10.1016/j.indcrop.2020.112931. 
Research, Society and Development, v. 10, n.12, e229101220227, 2021

Senhorini, G. A.; Zawadzki, S. F.; Farago, P. V.; Zanin, S. M. W. \& Marques, F. A. (2012). Microparticles of poly(hydroxybutyrate-co-hydroxyvalerate) loaded with andiroba oil: Preparation and characterization. Materials Science and Engineering C, 32, 1121-1126. https://dx.doi.org/10.1016/ j.msec.2012.02.027.

Sharif, K. M.; Rahman, M. M.; Azmir, J.; Mohamed, A.; Jahurul, M. H. A.; Sahena, F. \& Zaidul, I. S. M. (2014). Experimental design of supercritical fluid extraction - A review. Journal of Food Engineering, 124, 105-116. https://doi.org/10.1016/j.jfoodeng.2013.10.003.

Sharmin, E.; Zafar, F.; Akram, D.; Alam, M. \& Ahmad, S. (2015). Recent advances in vegetable oils based environment friendly coatings: a review. Ind. Crops Prod., 76, 215-222. https://doi.org/10.1016/j.indcrop.2015.06.022.

Silva, B. J. M.; Hage, A. A. P.; Silva, E. O. \& Rodrigues, A. P. D. (2018). Medicinal plants from the Brazilian Amazonian region and their antileishmanial activity: a review. Journal of Integrative Medicine, 16, 211-222. https://dx.doi.org/ 10.1016 / j.joim.2018.04.004.

Silva, J. A. P.; Cardoso, N. S. M. \& Petzhold, C. L. (2018). Enzymatic synthesis of andiroba oil based polyol for the production of flexible polyurethane foams. Industrial Crops \& Products, 113, 55-63. https://doi.org/10.1016/j.indcrop.2018.01.020.

Silva, J. J. M. \& Rogez, H. (2013). Avaliação da estabilidade oxidativa do óleo bruto de açaí (Euterpe oleracea) na presença de compostos fenólicos puros ou de extratos vegetais amazônicos. Química Nova, 36 (3), 400-406. https://doi.org/10.1590/S0100-40422013000300009.

Silva, R. P. F. F., Rocha-Santos, T. A. P. \& Duarte, A. C. (2016). Supercritical fluid extraction of bioactive compounds. TrAC Trends in Analytical Chemistry, 76, 40-51. https://doi.org/10.1016/j.trac.2015.11.013.

Solaesa, Á. G.; Sanz, M. T.; Falkeborg, M.; Beltrán, S., \& Guo, Z. (2016). Production and concentration of monoacylglycerols rich in omega-3 polyunsaturated fatty acids by enzymatic glycerolysis and molecular distillation. Food Chem., 190 , 960-967. http://dx.doi.org/10.1016/j.foodchem.2015.06.061.

Sousa, R. L.; Almeida, B. B.; Silva, R. P.; Albuquerque, L. C. S. \& Cordeiro, Y. E. M. (2019). Óleo de andiroba: extração, comercialização e usos tradicionais na comunidade Mamangal, Iguarapé-mirim, Pará. Biodiversidade, 10 (1), 68-81. https://periodicoscientificos.ufmt.br/ojs/index.php/biodiversidade/article/view

Xiong, J.; Matta, F. V.; Grace, M.; Lila, M. A.; Ward, N. I.; Sotelo, M. F. \& Esposito, D. (2020). Phenolic content, anti-inflammatory properties, and dermal wound repair properties of industrially processed and non-processed acai from the Brazilian Amazon. Food Function, 11 (6), 4903-4914. https://doi.org/ 10.1039/c9fo03109j.

Yamaguchi, K. K. L.; Pereira, L. F. R.; Lamarão, C. V.; Lima, E. S. \& Veiga-Junior, V. F. (2015). Amazon acai chemistry and biological activities: a review. Food Chemistry, 179, 137-151. https://doi.org/10.1016/j.foodchem.2015.01.055.

Yara-Varón, E.; Li, Y.; Balcells, M.; Canela-Garayoa, R.; Fabiano-Tixier, A. S. \& Chemat, F. (2017). Vegetable oils as alternative solvents for green oleoextraction, purification and formulation of food and natural products. Molecules, 22 (9), 1474, 1-24. https://dx.doi.org/10.3390/molecules22091474.

Yusoff, M. M.; Gordon, M. H. \& Niranjan, K. (2015). Aqueous enzyme assisted oil extraction from oilseeds and emulsion de-emulsifying methods: A review. Trends in Food Science \& Technology, 41 (1), 60-82. https://doi.org/10.1016/j.tifs.2014.09.003. 